\title{
INTERESTING SPATIO-TEMPORAL REGION DISCOVERY COMPUTATIONS OVER GPU AND MAPREDUCE PLATFORMS
}

\author{
Michael McDermott ${ }^{\mathrm{a}, *}$,Sushil K. Prasad ${ }^{\mathrm{b}}$, Shashi Shekhar ${ }^{\mathrm{c}}$, and Xun Zhou ${ }^{\mathrm{d}}$
}

\author{
aa Department of Computer Science, Georgia State University, USA - mmcdermott2@ student.gsu.edu \\ ${ }^{\mathrm{b}}$ Department of Computer Science, Georgia State University, USA - sprasad@ gsu.edu \\ ${ }^{c}$ Department of Computer Science, University of Minnesota, USA - shekhar@ cs.umn.edu \\ ${ }^{\mathrm{d}}$ Department of Management Sciences, University of Iowa, USA - xun-zhou @ uiowa.edu
}

KEY WORDS: GPU, MapReduce, Hadoop, Parallelism, GIS, Spatial, Spatio-Temporal, Data Mining

\begin{abstract}
:
Discovery of interesting paths and regions in spatio-temporal data sets is important to many fields such as the earth and atmospheric sciences, GIS, public safety and public health both as a goal and as a preliminary step in a larger series of computations. This discovery is usually an exhaustive procedure that quickly becomes extremely time consuming to perform using traditional paradigms and hardware and given the rapidly growing sizes of today's data sets is quickly outpacing the speed at which computational capacity is growing. In our previous work (Prasad et al., 2013a) we achieved a 50 times speedup over sequential using a single GPU. We were able to achieve near linear speedup over this result on interesting path discovery by using Apache Hadoop to distribute the workload across multiple GPU nodes. Leveraging the parallel architecture of GPUs we were able to drastically reduce the computation time of a 3-dimensional spatio-temporal interest region search on a single tile of normalized difference vegetative index for Saudi Arabia. We were further able to see an almost linear speedup in compute performance by distributing this workload across several GPUs with a simple MapReduce model. This increases the speed of processing 10 fold over the comparable sequential while simultaneously increasing the amount of data being processed by 384 fold. This allowed us to process the entirety of the selected data set instead of a constrained window.
\end{abstract}

\section{INTRODUCTION}

Interesting path and interesting spatio-temporal region discovery are important filtering steps in many domains such as earth and atmospheric sciences, GIS, public safety, public health and other fields which deal with identifying and analyzing spatial data that change over time. These are typically computationally expensive steps and can take hours to days to complete. For instance filtering non-interesting paths and regions is of particular interest when predicting weather patterns, designing accurate ecological models of climate shift and tracking ecotone ${ }^{1}$ boundaries and changes. Tracking these boundaries is a good way to study desertification, deforestation, erosion and other shifts in geographic areas which is of particular interest to GIS and environmental scientists.

Finding interesting paths and spatio-temporal regions are exhaustive operations. The reason for this is that the path or region does not necessarily have a clearly defined limit. In other words the interesting path or region could be as small as one item or as large as the whole data set (Zhou et al., 2011, Zhou et al., 2013). This leads to the less obvious, but important, consideration which is that any given interesting region or path may be contained in inside another larger region or path and this must be addressed somehow (Zhou et al., 2011 Zhou et al., 2013).

Data growth is also quickly outpacing our computational and management abilities on traditional hardware and algorithm implementations. More efficient ways of filtering and processing this data are important to develop in order to complete the computation in a reasonable time frame.

Parallel and distributed designs and implementations of these more efficient algorithms are also therefore becoming increasingly im-

\footnotetext{
* Corresponding author

${ }^{1}$ Ecotones are transitions between different ecologies such as the boundary between forest and grasslands (Zhou et al., 2011).
}

portant. Currently these sorts of big data calculations are limited to large clusters and supercomputers which are capable of processing the large volumes of data. Supercomputing environments are however expensive in time and effort to setup, manage and use. A much more accessible and economic approach is to equip a workstation with one or more inexpensive yet high performance GPU's which would allow rapid testing and deployment of new algorithms to solve problems in these domains. GPU hardware is economical both in terms of price to performance and in terms of power consumption to compute ability.

This paper's contributions are :

- To leverage GPU hardware to speed up discover of spatiotemporal regions of interest from an $O\left(M^{2} N^{2} T^{2}\right)$ computation to an $O\left(M N T^{2}\right)$ computation.

- To use a MapReduce model to distribute discovery of spatiotemporal regions of interest across multiple GPU devices which provides linear speedup with respect to the number of GPU devices used.

- To use Apache Hadoop to distribute interesting path discovery across multiple GPU compute nodes which provides a linear speedup over our previous work with respect to the number of nodes used.

- Sequential algorithms have problems processing large sizes of data (eg: the $4800 \times 4800$ map tiles discussed in Section 5 and have to limit computation to a smaller subset (eg: $200 \times 300$ ) in order to complete computation (Zhou et al., 2013). We have increased the size of the data that can be processed to the full map tile while simultaneously increasing performance for similar window sizes.

The remainder of this paper is organized as follows: In Section 2 the problems are introduced and discussed in a traditional sequential environment and algorithms introduced in previous works are outlined briefly. In Section 3 we briefly introduce and summarize our previous work on interesting path detection and talk about 
related works. In Section 4 we give an overview of the most important GPU considerations for this work and give a general design overview of our implementations of Hadoop+GPU subpath discovery, GPU ST-Region discovery and GPU MapReduce STRegion Discovery. In Section 5 we discuss the hardware environment used for our experiments. Section 6 shows our experimental results for Hadoop+GPU subpath discovery, GPU ST-Region discovery and GPU MapReduce ST-Region Discovery. Section 7 concludes the paper and gives some of our future research goals.

\section{PROBLEM DESCRIPTION}

In this work we are concerned with processing the large volumes of spatio-temporal data produced by remote sensing technologies frequently used in the study of climatology, oceanography, and geology. This data is generally stored in either vector or raster form. For our purposes we were interested in detecting paths and spatio-temporal regions of rapid change in normalized difference vegetative index data (NDVI) sets which are stored in raster image form ${ }^{2}$. These are frequently pre-processing steps used to limit the data being considered by much more costly computations and are also used to reveal interesting information in their own right. These problems are themselves computationally expensive as they must exhaustively consider the entire data set.

\subsection{Path Discovery}

Discovering interesting paths is the problem of discovering one dimensional longitudinal subpaths in a given raster data set that show increases (or decreases) in average NDVI value greater than a threshold value.

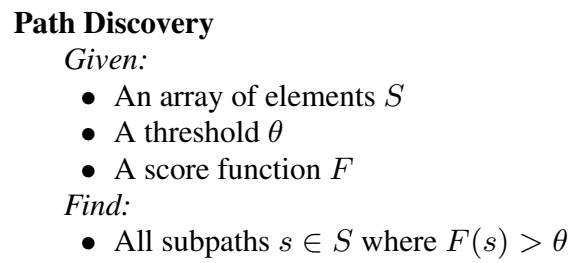

The determination of interestingness is described by Equation 1

$$
F(s)=\frac{\text { Average value of unit subpath }>\theta}{\text { Average value of all unit subpaths }}>\theta
$$

as in (Zhou et al., 2011, Prasad et al., 2013a).

Naively this test is trivial to implement but is computationally expensive with a cost of $O\left(N^{2} M\right)$ for an $N \times M$ tile of data. As we have to examine $N-1$ subpaths for every $N$ items and we must do this $M$ times. This quickly becomes problematic with today's large data sets.

(Zhou et al., 2011) provides an SEP algorithm which reduces computation by an order of magnitude over naive implementations through the construction of a lookup table in $O(N)$ time. This lookup table reduces an $O(N)$ scan operation to an $O(1)$ lookup, reducing the overall complexity to $O(N)+O\left(N^{2}\right)$.

The SEP algorithm has three phases:

1. Build the lookup table

2. Discover all interesting sub paths

${ }^{2}$ For more information on NDVI and these datasets see Section 5

\section{Eliminate paths which are subsets of longer paths}

The lookup table is a simple columnar prefix sum of the data in question. This can be done in $O(M N)$ time for spatial data of dimension $M x N$ (Zhou et al., 2011. Prasad et al., 2013a). Each column for the purposes of the discovery is assumed independent.

The discovery phase then uses the lookup table to find subpaths that pass the interestingness test. Longitudinal subpaths are preferred due to their being more likely to span ecotone boundaries (Zhou et al., 2011). This test is performed for each possible subpath in a given column and for each column of data starting at the largest possible subpath and examining each smaller subpath in turn until one is found that is interesting.

The elimination phase examines all discovered sub-paths and eliminates any that are completely enclosed inside a longer subpath.

\subsection{Spatio-temporal Region Discovery}

Discovering interesting spatio-temporal subregions is the problem of finding a two dimensional subregion that exhibits average change in NDVI value greater than a threshold value for a given interval of time.

\section{ST-Region Discovery \\ Given}

- A set of $R$ of $M x N$ regions organized by time

- A time interval to consider $T$

- A threshold $\Theta$

- A score function $F$

Find

- All sub-regions $r$ that are interesting

The interestingness test is described by Equation 2

$$
F(S)=\frac{\text { Average change in value for the subregion }}{\text { time interval }}
$$

As in (Zhou et al., 2013) this average change in value is defined as the value of the subregion at the beginning of the time interval minus the value of subregion at the end of the interval divided by the value of the beginning subregion. This is a measure of the average decrease in NDVI value. These beginning and ending values are determined by summing all the values in the subregion being considered. This must be done for both the beginning and the end of the interval being considered. This is illustrated in Figure 1 where the subregion at the beginning of the time interval is shown in blue and the corresponding subregion at the end of the time interval is shown in orange. The gray shaded region represents one of the possibly many intermediate subregions contained in the spatio-temporal volume being considered. For Figure 1 the time interval being considered is $n$ as it spans $n+1$ parent regions.

Naively this is also trivial to implement with the computational complexity increasing to $O\left(M^{4} N^{4} T^{2}\right)$ in the worst case. This is due to the need to exhaustively examine all possible spatiotemporal volumes. For small windows this computation time may be acceptable however in today's big data environment the time needed to perform this operation quickly becomes unacceptable as $M, N$ and $T$ become large.

(Zhou et al., 2013) provides a $P C W$ algorithm that mirrors the SEP algorithm's paradigms closely. The PCW algorithm reduces 


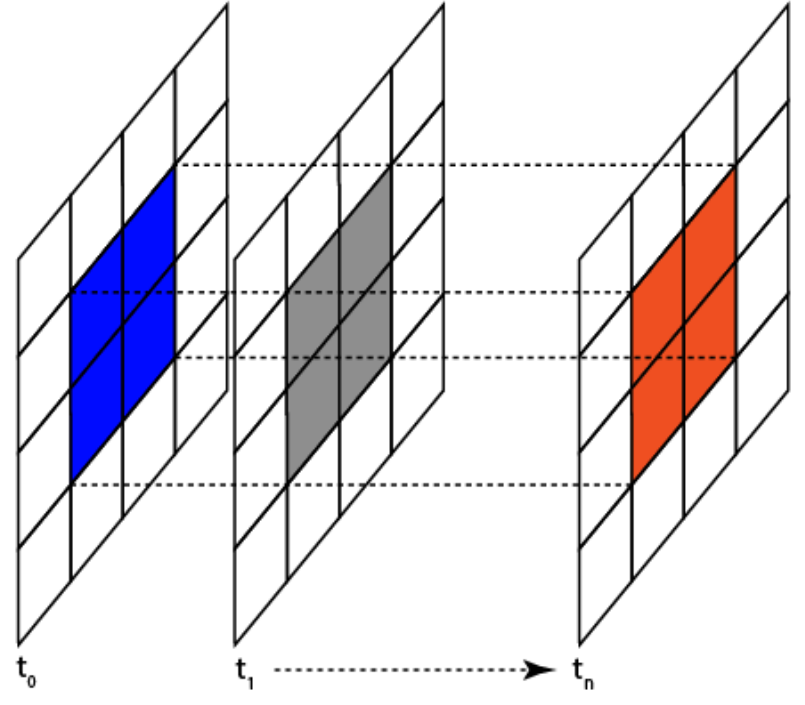

Figure 1. Subregion Discovery for $t_{0} \ldots t_{n}$

the worst case complexity to $O\left(M^{2} N^{2} T^{2}\right)$ by pre-computing a lookup table. This lookup table takes $O(M N)$ time and $O(M N)$ space in exchange for significantly reducing overall time complexity in a similar way to the SEP algorithm.

This algorithm also has three phases:

1. Build the lookup table

2. Discover all interesting subregions

3. Eliminate all subregions which are within larger regions

The discovery phase is also similarly scaled up in dimensions. The use of the lookup table is particularly important as it replaces an $O(N M T)$ scan for an $O(1)$ lookup. The elimination phase is also similarly scaled up and in this phase all PCW embedded in larger PCW are removed from results.

\section{PREVIOUS AND RELATED WORK}

\subsection{Previous Work}

In our previous work on interesting path discovery, the GPU implementation is straightforward due to the data independent nature of the problem (Prasad et al., 2013a). The lookup table is embarrassingly parallel to implement by launching a thread for every $n \in N$. By coalescing memory access the GPU is used efficiently to compute this scan very quickly. It should be noted that this is not truly a parallel scan but simply many smaller sequential scans launched in parallel. Due to the size of the data used for this work the performance impact was deemed to be negligible.

The subpath discovery is sped up significantly due also to the data independent nature of the problem. A worse case $O\left(N^{2} M\right)$ problem is reduced in theoretical complexity to $O(N M)$ through parallelization on the GPU (Prasad et al., 2013a).

The elimination step here was accomplished two ways; implicitly and explicitly. Implicit elimination allows larger subpaths to overwrite smaller ones when the data is visualized. Explicit elimination programatically removes smaller subpaths that are completely embedded in larger subpaths. The complexity of explicit elimination is the same as with discovery (Prasad et al., 2013a).

Overall this resulted in a good speedup and the ability to process a single image in the data set in roughly $30 \mathrm{~ms}$. We were able to process the entire data set quickly enough to generate real-time visualizations of the algorithm's output on a single GPU (Prasad et al., 2013b. Prasad et al., 2013a). We use this data set and GPU implementation in Section 4.1

\subsection{Related Work}

MapReduce is a high level data centric model of distributed computing that only requires two main phases; the map phase to distribute data and the reduce phase to perform computation and return results.

There are currently few related works dealing with MapReduce and GPU. The first is Mars, a GPU MapReduce framework implemented on GPUs in $\mathrm{C} / \mathrm{C}++$ and Cuda (He et al., 2008). This uses a simplified version of MapReduce which only has two phases, map and reduce. The second is GPMR, a GPU MapReduce library geared towards GPU clusters with scalability as a specific concern (Stuart and Owens, 2011). Self contained and extensible, the GPU is still exposed to the user in contrast to Mars which sought to obfuscate the GPU behind its own MapReduce interface. The third, StreamMR, is a GPU MapReduce framework based on OpenCL and designed for clusters of AMD GPUs (Elteir et al., 2011).

There are two main works that deal specifically with MapReduce and spatial data: Spatial-Hadoop and HadoopGIS. SpatialHadoop is an extension of Hadoop that provides access to spacial primitives and common spatial operations (Eldawy, 2014). HadoopGIS is a spatial data query system for performing spatial queries in Hadoop (Aji et al., 2013). However, neither SpatialHadoop nor HadoopGIS have built-in support for GPU integration currently.

\section{DESIGN AND IMPLEMENTATION DETAILS}

In order to achieve the best performance from the GPU hardware some general guidelines and best practices must be followed.

CUDA General Guidelines

- Minimize branching

- Minimize global memory access

- Maximize global memory bandwidth utilization

Minimizing branching has to do with the GPU being a single instruction multiple data (SIMD) architecture. Threads in a block are grouped together into warps of up to 32 threads with threads within a warp ideally executing the same instruction. When there is branching within a warp there is the potential to halve the performance of the warp. With enough branching this can reduce performance to near sequential time.

Minimizing global memory access and maximizing global memory bandwidth utilization are closely related. Global memory, the large memory measured in gigabytes on the GPU device, has very slow access time compared to the local thread memory and shared block memory which are much smaller. When accessing global memory, for maximum performance, it must be accessed in a thread aligned way. This means that threads in the same warp access contiguous portions of global memory in order to speed up access and maximize throughput (NVIDIA, 2013).

The GPU implementation utilizes the ideas of the SEP and PCW algorithm, however the algorithm implementations have been reengineered with the above considerations in mind. 


\subsection{GPU+Hadoop Path Discovery}

Using the single GPU implementation from our previous work (Prasad et al., 2013a), the Hadoop implementation is fairly straightforward. At the high level:

1. Raw raster data is read into a sequence file on and saved on the HDFS.

2. Values are mapped from the sequence file.

3. GPU computation is performed on compute nodes in the reduce phase.

GPU kernels were written in CUDA and then launched by Hadoop using the JCUDA ${ }^{3}$ Java bindings library. This allowed quick deployment to Hadoop once the GPU code had been developed.

This also means that care must be taken in the map phase so that the data is not subdivided incorrectly. We used a sequence file to guarantee that this did not happen. This also allowed us to keep the data in a binary format (White, 2009). Using the sequence file allowed avoidance of the small files problem as we were able to read in all the relatively small raster data files and combine them into one large file of key, value pairs. For simplicity each raster data file was read in as a single value in the sequence file.

The small file problem stems from how the HDFS maps files. Each file in HDFS uses at least one HDFS block. For files larger than this block size this is generally not an issue. When files are smaller than the block size then space in the HDFS is wasted. This can potentially result in decreased performance of the HDFS as a whole by increasing file read and write (White, 2009).

\subsection{GPU Spatio-temporal Region Discovery}

The previous paradigm for building the looking table, while simple, is comparatively inefficient. A better strategy is a GPU implementation of parallel prefix sum due to the size of the data set. This algorithm is adapted from the segmented sums algorithm of (Nguyen, 2007) and is sketched briefly in Algorithm 1 This algorithm suffers from the limitation that it cannot perform a prefix sum on an array larger than the maximum threads allowed in a block. In order to overcome this limitation a segmented sum is used. The last element of each prefix summed block of threads is used to populate a new array which is then prefix summed with the same algorithm and limitations. This is done as a reduction tree until a single block of threads can process the array. These values are then back-propagated up through the reduction tree in parallel. This back-propagation step can be seen in Figure 2 .

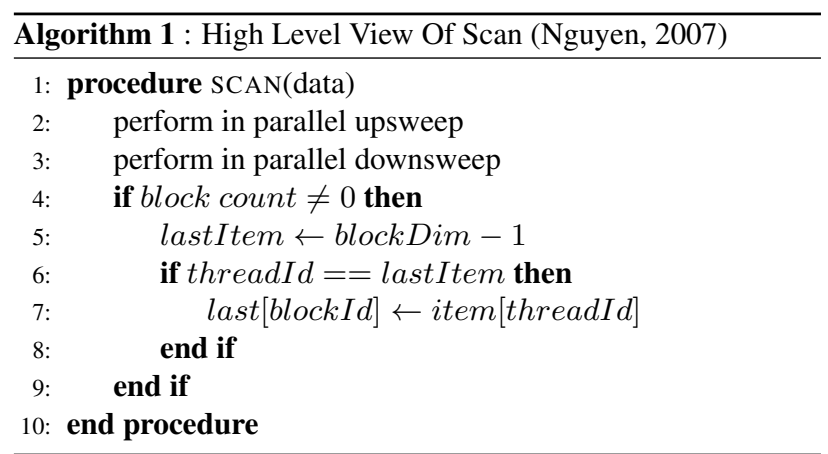

This scan is complex and requires multiple kernel invocations but it leverages the strengths of the GPU extremely well. The overall complexity for construction of the table is $O(N \log M)$.

\footnotetext{
${ }^{3}$ For more information see http: //www.jcuda.org
}

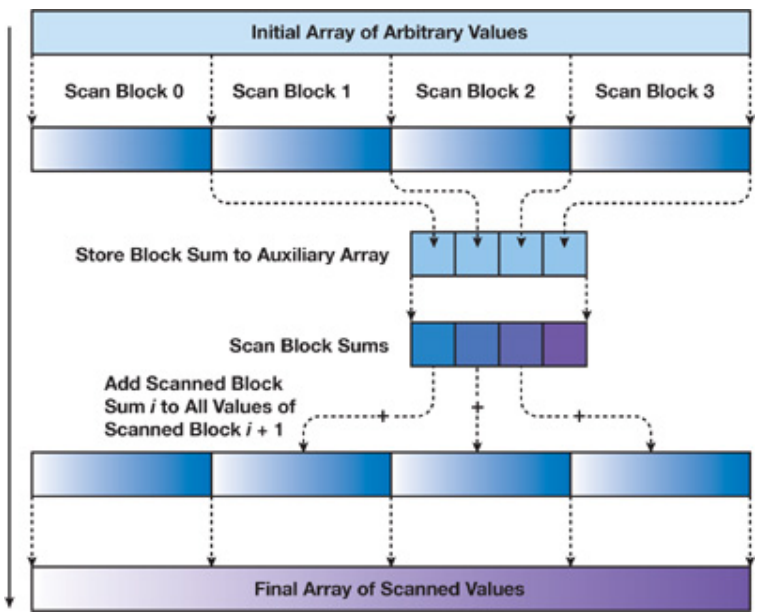

Figure 2. Illustration of segmented scan propagation (Nguyen, 2007)

The PCW algorithm is straightforward to parallelize due to the relative independence of each subregion being considered. It is performed in parallel with a thread for each starting position. This reduces the complexity to a theoretical $O\left(M N T^{2}\right)$ computation when $M N$ threads are used. An $O(1)$ lookup is performed in order to retrieve the subregion values needed for Equation 2

This lookup is described by Equation 3

$$
\sum \text { subregion value }=\sum X-\sum Y-\sum Z+\sum U
$$

Where $\mathrm{X}, \mathrm{Y}, \mathrm{Z}$, and $\mathrm{U}$ are the prefix sums at the locations shown in Figure 3 and we want the value associated with the subregion described by the shaded portion of Figure 3

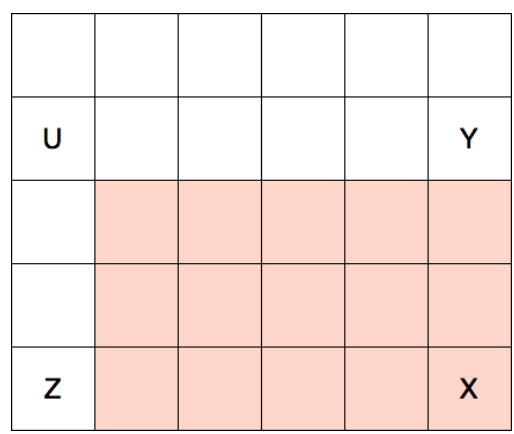

Figure 3. Illustration of lookup table

This is an efficient operation and widely used in many computer graphics domains (Crow, 1984, Zhou et al., 2013).

The implementation details of the algorithm require significant re-engineering in order to reduce branching to a minimum, maximize memory bandwidth, coalesce memory access and ensure that blocks of threads never consider areas that are outside the dimensions of the data set. The high level algorithm sketch for a thread block is shown in Algorithm 2

Each block of threads retrieves start locations and then iteratively retrieves possible end locations and performs this discovery test. When the discovery test is finished the block gets the next block sized subset of end locations.

The elimination phase for ST-Region discovery is not included in this paper and is left for future work due to time considerations. 


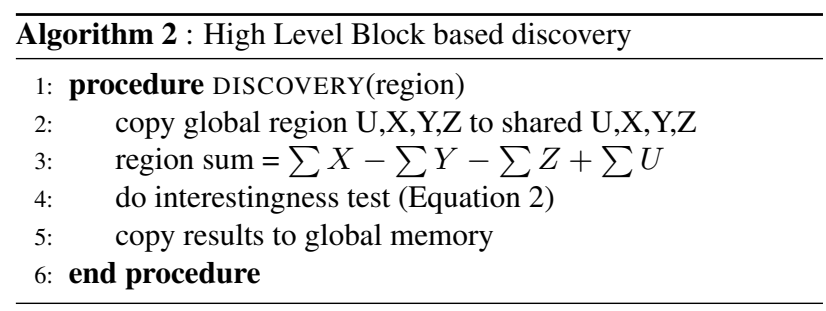

\subsection{Multiple-GPU MapReduce Spatio-Temporal Region Dis- covery}

The multiple GPU MapReduce model is straightforward due to the overall design of the GPU implementation being itself a MapReduce model. Each time interval calculation is also independent for the interestingness test and easily maps to a distinct GPU. For instance the calculations needed for interval $t_{0} \ldots t_{i}$ are independent of those needed for interval $t_{0} \ldots t_{j}$. Each GPU also has its own unique device ID, making it easy to iterate through the time intervals and assign an interval to a specific device. It is straightforward to do this assignment, computation, and memory transfer asynchronously (NVIDIA, 2012), allowing the devices to run independently of each other and the CPU. As each device finishes a time interval it reduces the output back to the CPU asynchronously then it immediately starts on the next interval it has been mapped.

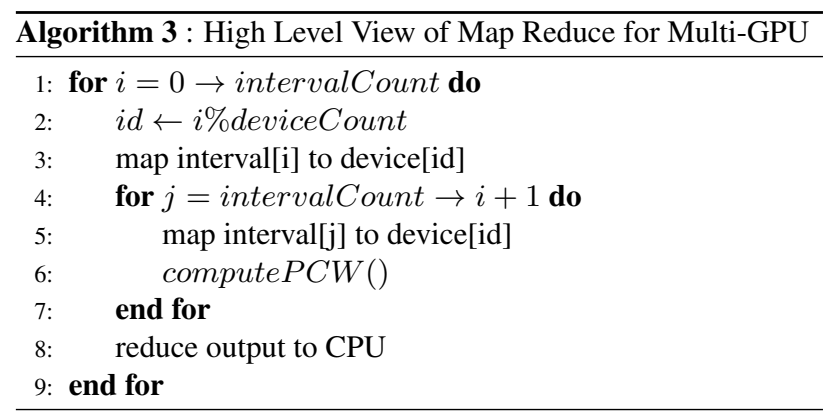

\section{DATA AND HARDWARE ENVIRONMENT}

The datasets used are normalized difference vegetative index data (NDVI); a measure of vegetative density. NDVI is computed by measuring reflectance of specific wavelengths of light off the surface of the earth through remote sensing (Weier and Herring, 2000).

\subsection{GPU+Hadoop Path Discovery}

GPU+Hadoop Path discovery was performed using a heterogeneous cluster environment and the same data set as in our previous work. This cluster consists of several nodes equipped with NVIDIA GTX 480 and NVIDIA Tesla C2075 GPUs. The data set used is the Global Inventory Modeling and Mapping Studies (GIMMS) data set which consists of 20 years of NDVI resulting in 611 images of Africa of size $1152 \times 1152$ (Tucker and Brown, 2004). This data set was used in order to do a fair comparison between the GPU, Hadoop with GPU, and sequential environments. Nodes are equiped with memory ranging from $8 \mathrm{~GB}$ to $64 \mathrm{~GB}$ of memory and either dual Intel Xeon Quad Core 5410 or dual Intel Xeon E5-2650 CPUs.

\subsection{Spatio-Temporal Region discovery}

Spatio-Temporal Region discovery was performed using NVIDIA Tesla k20 GPUs. The data set used was a subset of NDVI data taken from the Land Processes Distributed Active Archive Center (LPDAAC) and consists of 12 years, 2000 through 2012, of $4800 \times 4800$ NDVI data selected from the NASA MODIS project (NASA and USGS, 2015). As stated in (Zhou et al., 2013) the sequential discovery algorithm was limited to a small subsection of this data set $(200 \times 300)$, however, on the GPU we processed the entire $4800 \times 4800$ map tile. MapReduce was implemented on a single compute node that housed four Tesla $\mathrm{k} 20 \mathrm{GPUs}$. This node is equipped with 64GB of memory and dual Intel Xeon E5-2650 CPUs.

\section{EXPERIMENTAL RESULTS}

Initial results are very promising and significant performance increases were achieved by porting to GPU and Multi-GPU environments.

\subsection{GPU+Hadoop Path Discovery}

The Hadoop implementation resulted in a nearly linear speedup with the addition of more nodes as can be seen in Figure 4 but also made realizing real-time visualization impossible. There are diminishing returns as more nodes are added. It is expected that this is because of the data size being relatively small at only roughly $300 \mathrm{MB}$ for the entire 611 NDVI images.

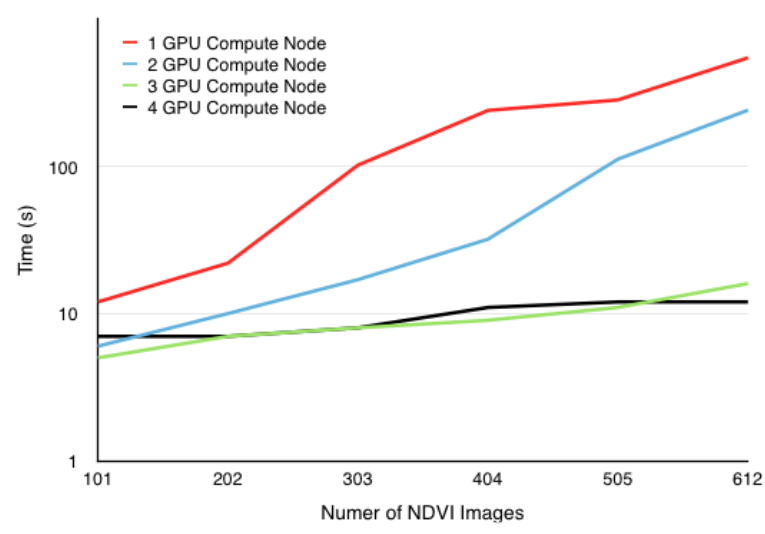

Figure 4. Path Discovery Hadoop Scaling

\subsection{Spatio-Temporal Region Discovery}

Compute speeds increased 50 fold over naive sequential, which was expected due to performance increases seen for the sequential SEP algorithm. Compute speeds increased 10 fold compared to SEP algorithm. These speedups do not at first seem impressive however it must be kept in mind that the largest PCW processed sequentially in (Zhou et al., 2013) was a $50 \times 50$ subregion on a very limited subwindow of $200 \times 300$; no such artificial constraint on window size was enforced on the GPU implementation and we processed the entire raster tile exhaustively.

This means the realized speedup is comparing computation on a $200 \times 300$ window to computation on a $4800 \times 4800$ window. In other words we realized a good speedup and simultaneously were able to increase the maximum potential window size by 384 fold.

Time interval size is held constant at $T=10$ while window dimensions are increased in Figure 5 Starting with a window of $50 \times 50$ we processed the entire $4800 \times 4800$ MODIS map tile data set in 193.83 seconds on a single GPU. Utilizing four GPUs and a MapReduce model reduces this time to roughly $52.48 \mathrm{sec}-$ onds. 


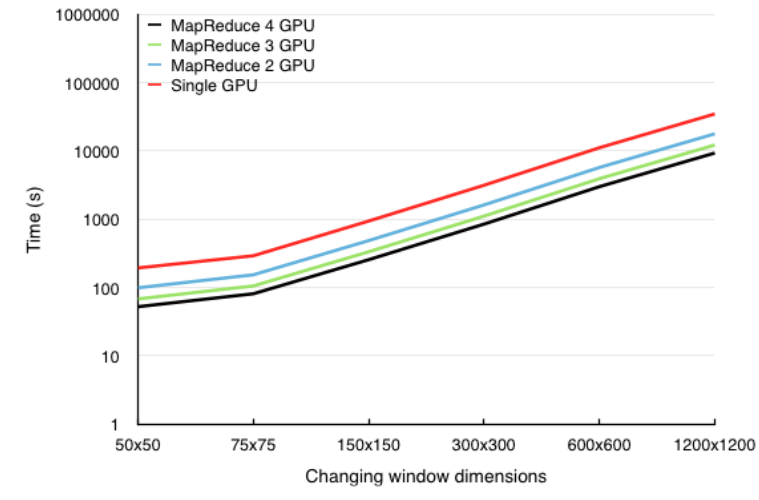

Figure 5. ST-Region GPU MapReduce timing with varying window dimension (time interval $T=10$ )

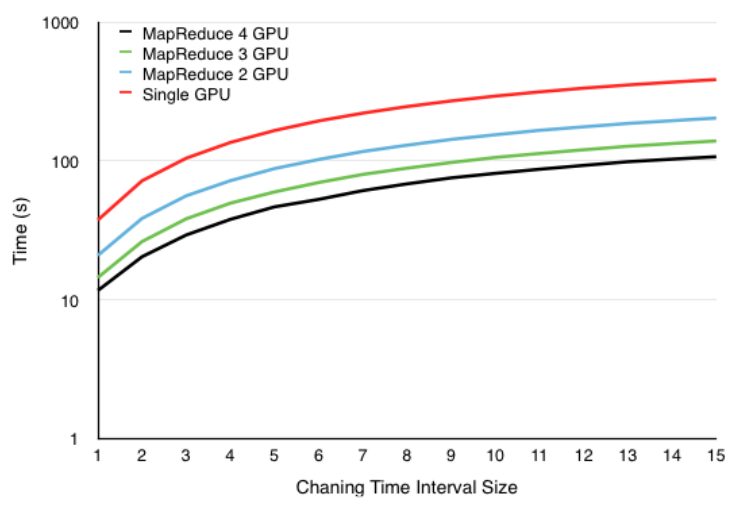

Figure 6. ST-Region GPU MapReduce with varying Time interval size $($ fixed dimension $=75 \times 75)$

Holding the PCW dimensions fixed at $75 \times 75$ we can see the effects here of varying the time interval in Figure 6

Both plots demonstrate how scaling is affected by varying dimensions. Adding GPUs has diminishing returns for small query sizes, however the scaling approaches linear for the larger queries. This reduces total time taken by a factor equal to the number of GPUs used.

\section{CONCLUSION AND FUTURE WORK}

This work explores an implementation of the SEP and PCW algorithms from (Zhou et al., 2011, Zhou et al., 2013) that clearly show an increase not just in execution time but in the amount of data that can be processed. While valuable in its own right this is also a valuable consideration for what may be a preprocessing step for a much larger and more intensive computational model. Offloading this processing onto the GPU, while more time consuming in the development phase, is a viable technique to speed up these calculations. This will become increasingly more important to achieve as big data considerations continue to become more and more important to address.

Future works include a GPU and GPU+MapReduce model for the elimination phase in the PCW algorithm as well as processing multiple tiles.

\section{REFERENCES}

Aji, A., Wang, F., Vo, H., Lee, R., Liu, Q., Zhang, X. and Saltz, J., 2013. Hadoop gis: A high performance spatial data warehousing system over mapreduce. Proc. VLDB Endow. 6(11), pp. 10091020.

Crow, F. C., 1984. Summed-area tables for texture mapping. SIGGRAPH Comput. Graph. 18(3), pp. 207-212.

Eldawy, A., 2014. Spatialhadoop: Towards flexible and scalable spatial processing using mapreduce. In: Proceedings of the 2014 SIGMOD PhD Symposium, SIGMOD'14 PhD Symposium, ACM, New York, NY, USA, pp. 46-50.

Elteir, M., Lin, H., Feng, W.-c. and Scogland, T., 2011. Streammr: An optimized mapreduce framework for amd gpus. In: Proceedings of the 2011 IEEE 17th International Conference on Parallel and Distributed Systems, ICPADS '11, IEEE Computer Society, Washington, DC, USA, pp. 364-371.

He, B., Fang, W., Luo, Q., Govindaraju, N. K. and Wang, T., 2008. Mars: A mapreduce framework on graphics processors. In: Proceedings of the 17th International Conference on Parallel Architectures and Compilation Techniques, PACT '08, ACM, New York, NY, USA, pp. 260-269.

NASA and USGS, 2015. http://lpdaac.usgs.gov/ products/modis_products_table/mod13q1

Nguyen, H., 2007. Gpu Gems 3. First edn, Addison-Wesley Professional.

NVIDIA, 2012. http://devblogs.nvidia.com/ parallelforall/how-overlap-data-transfers-cuda$\mathrm{cc} /$.

NVIDIA, 2013. http://devblogs.nvidia.com/ parallelforall/how-access-global-memoryefficiently-cuda-c-kernels/

Prasad, S. K., Shekhar, S., McDermott, M., Zhou, X., Evans, M. and Puri, S., 2013a. GPGPU-accelerated interesting interval discovery and other computations on geospatial datasets: A summary of results. In: Proceedings of the 2Nd ACM SIGSPATIAL International Workshop on Analytics for Big Geospatial Data, BigSpatial '13, ACM, New York, NY, USA, pp. 65-72.

Prasad, S., Shekhar, S., He, X., Puri, S., McDermott, M., Zhou, X. and Evans, M., 2013b. Gpgpu-based data structures and algorithms for geospatial computation a summary of results and future roadmap (position paper). Proceedings of The All Hands Meeting of the NSF CyberGIS project, Seattle.

Stuart, J. A. and Owens, J. D., 2011. Multi-gpu mapreduce on gpu clusters. In: Proceedings of the 2011 IEEE International Parallel \& Distributed Processing Symposium, IPDPS '11, IEEE Computer Society, Washington, DC, USA, pp. 1068-1079.

Tucker, C.J., J. P. and Brown, M., 2004. Global Inventory Modeling and Mapping Studies, NA94apr15b.n11-VIg, 2.0. Global Land Cover Facility, University of Maryland, College Park, Maryland.

Weier, J. and Herring, D., 2000. Measuring vegetation (ndvi \& evi). http://earthobservatory.nasa.gov/Features/ MeasuringVegetation/

White, T., 2009. Hadoop: The Definitive Guide. 1st edn, O’Reilly Media, Inc.

Zhou, X., Shekhar, S. and Oliver, D., 2013. Discovering persistent change windows in spatiotemporal datasets: A summary of results. In: Proceedings of the 2nd ACM SIGSPATIAL International Workshop on Analytics for Big Geospatial Data, BigSpatial '13, ACM, New York, NY, USA, pp. 37-46. 
Zhou, X., Shekhar, S., Mohan, P., Liess, S. and Snyder, P. K., 2011. Discovering interesting sub-paths in spatiotemporal datasets: A summary of results. In: Proceedings of the 19th ACM SIGSPATIAL International Conference on Advances in Geographic Information Systems, GIS '11, ACM, New York, NY, USA, pp. 44-53. 